\title{
THE APPROXIMATION ORDER OF BOX SPLINE SPACES
}

\author{
A. RON AND N. SIVAKUMAR
}

(Communicated by J. Marshall Ash)

Dedicated to Professor I. J. Schoenberg, in memoriam

\begin{abstract}
Let $M$ be a box spline associated with an arbitrary set of directions and suppose that $S(M)$ is the space spanned by the integer translates of $M$. In this note, the subspace of all polynomials in $S(M)$ is shown to be the joint kernal of a certain collection of homogeneous differential operators with constant coefficients. The approximation order from the dilates of $S(M)$ to smooth functions is thereby characterized. This extends a well-known result of de Boor and Höllig (B-splines from parallelepipeds, J. Analyse Math. 42 (1982/83), 99-115), on box splines with integral direction sets.

The argument used is based on a new relation, valid for any compactly supported distribution $\phi$, between the semidiscrete convolution $\phi *^{\prime}$ and the distributional convolution $\phi *$.
\end{abstract}

\section{INTRODUCTION}

Let $\Xi$ be a real $s \times n$ matrix with nonzero columns. At times we think of $\Xi$ as the collection of its column vectors, so that $\xi \in \Xi$ means that $\xi$ is a column of $\Xi$ and $Y \subset \Xi$ means that $Y$ is an $s \times k(k \leq n)$ submatrix of $\Xi$. The box spline $M \Xi$ associated with $\Xi$ is defined to be the Dirac distribution in case $\Xi$ is empty (i.e., $n=0$ ), and otherwise by the distributional rule

$$
M_{\Xi}(\psi):=\int_{[0,1]^{n}} \psi(\Xi t) d t, \quad \forall \psi \in C\left(\mathbb{R}^{s}\right) .
$$

Its Fourier transform is given by

$$
\widehat{M_{\Xi}}(w)=\prod_{\xi \in \Xi} \int_{0}^{1} e^{-i w \cdot \xi t} d t .
$$

In general, $M_{\Xi}$ can be identified with a positive measure supported on a compact polyhedral subset of the column span of $\Xi$, and, in case $\Xi$ is of rank

Received by the editors July 26, 1990 and, in revised form, June 17, 1991; presented at the AMS Regional Conference, Tampa, FL, March, 1991.

1991 Mathematics Subject Classification. Primary 41A15, 41A63, 41A25.

Key words and phrases. Box splines, polynomials, multivariate splines, approximation order.

The first author was supported in part by the United States Army under Contract No. DAAL0387-K-0030 and by the National Science Foundation under Grant No. DMS-9000053. The second author was partially sponsored by the Faculty of Graduate Studies and Research and NSERC Grant \# A7687. 
$s, M_{\Xi}: \mathbb{R}^{s} \rightarrow \mathbb{R}_{+}$is a compactly supported piecewise polynomial function. Various specific relevant references on box splines are given in the sequel. For expository material on box splines, we refer the reader to [C] (and references therein) as well as to the forthcoming book of de Boor, Höllig, and Riemenschneider [BHR].

The main purpose of this note is to characterize the approximation order of box spline spaces. For any compactly supported distribution $\phi$, we define $S(\phi)$ to be the (infinite) span of the integer translates of $\phi$ :

$$
S(\phi):=\operatorname{span}\left\{E^{\alpha} \phi: \alpha \in \mathbb{Z}^{s}\right\},
$$

with $E^{x}, x \in \mathbb{R}^{s}$, the translation operator,

$$
E^{x}: f \mapsto f(\cdot+x)
$$

A space of the form $S(M)$, for a box spline $M$, is referred to as a box spline space. To define the approximation order of $S(M)$, we need a way to refine this space. A refinement $S_{h}(\phi)$ (with $h$ positive and small) of $S(\phi)$ can be obtained by scaling $S(\phi)$,

$$
S_{h}(\phi):=\{f(\cdot / h): f \in S(\phi)\} .
$$

The approximation order of $S(\phi)$ (in the $\infty$-norm) is then the maximal integer $d$ that satisfies

$$
\operatorname{dist}_{\infty}\left(f, S_{h}(\phi)\right)=O\left(h^{d}\right), \quad \forall f \in W_{\infty}^{d},
$$

where $W_{\infty}^{d}$ is the usual Sobolev space. For a function $\phi$, the study of approximation orders for the space $S(\phi)$ is significantly facilitated by the Strang-Fix Conditions. These conditions focus on the space $\Pi(\phi)$ of all polynomials in $S(\phi)$ and assert (cf. [R]) that the approximation order of $S(\phi)$ is the maximal integer $d$ for which

$$
\Pi_{d-1} \subset \Pi(\phi)
$$

provided that $\hat{\phi}(0) \neq 0$. (Here and elsewhere $\Pi:=\Pi\left(\mathbb{R}^{s}\right)$ is the space of all complex-valued $s$-variate polynomials and $\Pi_{k}$ is the subspace of all polynomials of total degree at most $k$.) Consequently, the question of approximation orders is reduced to the identification of the space $\Pi(\phi)$.

A characterization of $\Pi\left(M_{\Xi}\right)$ is well known in case $\Xi$ is an integral matrix (i.e., all entries in $\Xi$ are integers). To describe it, we associate with each column $\xi$ of $\Xi$ the polynomial $p_{\xi}: x \mapsto \xi \cdot x$, and define

$$
p_{Y}:=\prod_{\xi \in Y} p_{\xi}, \quad Y \subset \Xi
$$

Thus $p_{Y}(D)$ is the product of the directional derivatives $p_{\xi}(D)$ (in any order). With

$$
\mathbb{K}(\Xi):=\{Y \subset \Xi: \operatorname{rank}(\Xi \backslash Y)<s\}
$$

it is known that

$$
\Pi\left(M_{\Xi}\right)=D(\Xi):=\left\{q \in \Pi: p_{Y}(D) q=0, \forall Y \in \mathbb{K}(\Xi)\right\}
$$


Since $\Pi\left(M_{\Xi}\right)$ is obtained in (1.10) as the intersection (in $\Pi$, but as a matter of fact even in the distribution space $\mathscr{D}^{\prime}\left(\mathbb{R}^{s}\right)$ ) of kernels of homogeneous differential operators with constant coefficients, it follows that

$$
\Pi_{d-1} \subset \Pi\left(M_{\Xi}\right) \Leftrightarrow(\# Y \geq d, \forall Y \in \mathbb{K}(\Xi)),
$$

where $\# Y$ is the number of columns in the matrix $Y$, i.e., the cardinality of the multiset $Y$. Consequently, the approximation order of $S\left(M_{\Xi}\right)$ is the number

$$
d_{\Xi}:=\min \{\# Y: Y \in \mathbb{K}(\Xi)\},
$$

i.e., the lowest degree of the differential operators involved in the definition of $D(\Xi)$. These results were first established by de Boor and Höllig in $[\mathrm{BH}]$ and were also proved (with the aid of different arguments and in a slightly more general setting) by Dahmen and Micchelli in [DM].

As emphasized earlier, this characterization of the approximation order for $S\left(M_{\Xi}\right)$ is valid only when the underlying matrix $\Xi$ is integral. For a general $\Xi$, it is still true $[\mathrm{BH}]$ that $M_{\Xi}$ is piecewise in $D(\Xi)$, so that $\Pi\left(M_{\Xi}\right) \subset D(\Xi)$ and the number $d_{\Xi}$ given in (1.12) provides an upper bound for the approximation order of $S\left(M_{\Xi}\right)$. Yet, simple examples show that in general this bound is not attained and may be far from the actual approximation order. In this note we show that, surprisingly, $\Pi\left(M_{\Xi}\right)$ is always realizable as the common nullspace of certain differential operators of the form $p_{Y}(D), Y \subset \Xi$. We thereby extend the aforementioned results of [BH, DM] to nonintegral matrices.

Our argument is based on the interplay between the convolution operator $M_{\Xi *}$ and related differential and difference operators [BH]. To make this interplay effective in the setting here, we invoke, in $\S 2$, the Poisson summation formula in a way that reduces the characterization of $\Pi(\phi)$ (for a compactly supported distribution $\phi)$, to the study of the action of the convolution operator $\phi *$ on the exponential spaces $e_{\alpha} \Pi, \alpha \in 2 \pi \mathbb{Z}^{s}$ (henceforth, $e_{\alpha}(\cdot)=e^{i \alpha^{\bullet}}$ ). This avoids the standard conversion of the problem into the Fourier transform domain. The main result is stated and proved in $\S 3$ and is followed by some discussion and examples.

\section{SeMidisCRETE CONVOLUtion}

Throughout this paper $\phi$ is assumed to be a compactly supported distribution (in $s$ dimensions). We reserve the notation $\phi *$ for the standard distributional convolution operator (defined on $\mathscr{D}^{\prime}$ ), and, following [B], use the notation $\phi *^{\prime}$ for the semidiscrete convolution operator that is defined as

$$
\phi *^{\prime}: f \mapsto \phi *^{\prime} f:=\sum_{\alpha \in \mathbb{Z}^{s}} f(\alpha) E^{-\alpha} \phi \in S(\phi),
$$

with $f$ being any function defined (at least) on $\mathbb{Z}^{s}$.

The following result is useful in the study of $\Pi(\phi)$. For a function $\phi$ it can be found in [B].

Proposition 2.2. Assume that $\phi *$ is $1-1$ on $\Pi$. Then

$$
\Pi(\phi)=\left\{p \in \Pi: \phi *^{\prime} p \in \Pi\right\}=\phi *^{\prime} \Pi(\phi) .
$$

Proof. Since $\Pi(\phi)$ is translation invariant [B], it is an invariant space of $\phi *$; hence, the injectivity of $\phi *$ on $\Pi$ implies that $\phi * \Pi(\phi)=\Pi(\phi)$. Set

$$
Q:=\left\{p \in \Pi: \phi *^{\prime} p \in \Pi\right\} \text {. }
$$


By [BR1], $\phi *^{\prime} Q=\Pi(\phi)$, while, by [BR2],

$$
\phi *^{\prime} p \in \Pi \Leftrightarrow \phi *^{\prime} p=\phi * p, \quad p \in \Pi \text {. }
$$

Thus $\phi * Q=\Pi(\phi)$. Since we have also shown that $\phi * \Pi(\phi)=\Pi(\phi)$, it follows that $Q=\Pi(\phi)$, and the proof is complete.

At this juncture, we wish to point out that $\phi * \Pi \subseteq \Pi$ and that the injectivity of $\phi *$ on $\Pi$ is equivalent to the statement $\hat{\phi}(0) \neq 0$. To see this equivalence, note that on the one hand, if $\hat{\phi}(0)=0$ then $\widehat{\phi * 1}=\hat{\phi} \delta=0$, hence $\phi * 1=0$, i.e., $\phi *$ is not injective on $\Pi$. Conversely, if $\hat{\phi}(0) \neq 0$ then $\phi * 1 \neq 0$, so $\phi *$ is degree-preserving and consequently injective on $\Pi$.

Proposition 2.2 suggests the study of the map $\phi *^{\prime}{ }_{\mid \Pi}$ as a means of identifying the space $\Pi(\phi)$. It is therefore rather annoying to realize that, in contrast with the standard convolution operator $\phi *, \phi *^{\prime}$ does not commute with nonintegral translations and hence fails to commute with differentiation. This obstacle is being circumvented here with the aid of the identity

$$
\phi *^{\prime} f=\sum_{\alpha \in 2 \pi \mathbb{Z}^{s}} \phi *\left(e_{\alpha} f\right),
$$

which is valid under various conditions on the pair $(\phi, f)$. It is a direct application of Poisson's summation formula, as in [SF, DM, B, BR2], but, unlike these cited references, is not restricted to a polynomial or an exponential $f$. More importantly, it does not convert the problem into the Fourier transform domain, thus allowing us to exploit efficiently the favourable properties of the convolution operator $\phi *$.

Theorem 2.6. Let $\phi$ be a compactly supported distribution and $f$ an infinitely differentiable function. Then $\phi *^{\prime} f$ and $\sum_{\alpha \in 2 \pi \mathbb{Z}^{s}} \phi *\left(e_{\alpha} f\right)$ converge in $\mathscr{D}^{\prime}$ and to the same limit, i.e., (2.5) holds.

Proof. By Poisson's summation formula [F, p. 104], the sum $\sum_{\alpha \in 2 \pi \mathbb{Z}^{s}} e_{\alpha}$ converges in $\mathscr{D}^{\prime}$ to $\sum_{\alpha \in \mathbb{Z}^{s}} \delta_{\alpha}$ (with $\delta_{\alpha}$ being point-evaluation at $\alpha$ ). Thus, since multiplication by $f \in C^{\infty}$ as well as convolution with a compactly supported $\phi$ are continuous operations in $\mathscr{D}^{\prime}$, we obtain

$$
\begin{aligned}
\phi *^{\prime} f & =\sum_{\alpha \in \mathbb{Z}^{s}} f(\alpha) \phi * \delta_{\alpha}=\sum_{\alpha \in \mathbb{Z}^{s}} \phi *\left(f \delta_{\alpha}\right)=\phi *\left(f \sum_{\alpha \in \mathbb{Z}^{s}} \delta_{\alpha}\right) \\
& =\phi *\left(f \sum_{\alpha \in 2 \pi \mathbb{Z}^{s}} e_{\alpha}\right)=\phi *\left(\sum_{\alpha \in 2 \pi \mathbb{Z}^{s}} e_{\alpha} f\right)=\sum_{\alpha \in 2 \pi \mathbb{Z}^{s}} \phi *\left(e_{\alpha} f\right) .
\end{aligned}
$$

Remark 2.7. Since the convergence of the sum $\sum_{\alpha \in 2 \pi \mathbb{Z}^{s}} e_{\alpha}$ holds in much stronger topologies than the $\mathscr{D}^{\prime}$-topology, the smoothness assumption on $f$ can be relaxed. Further, upon restricting the growth rate of $f$ at $\infty$, we may allow $\phi$ to have noncompact support. However, the simple form above of Theorem 2.6 is sufficient for all the applications considered in this paper, if, in addition, we observe that for a function $f \in O_{M}$ (i.e., $f$ is infinitely smooth and has, along with all its derivatives, polynomial growth at $\infty$ ), equation (2.5) extends to $\mathscr{S}^{\prime}$.

Indeed, our subsequent application concerns the special case when $(\phi$ is compactly supported and) $f$ is a polynomial. In this case, Theorem 2.6 leads 
to the following corollary, the first part of which was previously established in [BR2].

Corollary 2.8. For a compactly support $\phi$ and a polynomial $p$,

$$
\phi *^{\prime} p \in \Pi \Leftrightarrow \phi *^{\prime} p=\phi * p \Leftrightarrow \phi *\left(e_{\alpha} p\right)=0, \quad \forall \alpha \in 2 \pi \mathbb{Z}^{s} \backslash 0 .
$$

Proof. By Theorem 2.6 and Remark 2.7, $\phi *^{\prime} p-\phi * p$ has its Fourier transform supported on $2 \pi \mathbb{Z}^{s} \backslash 0$, hence can never be a nontrivial polynomial, while obviously, $\phi * p$ is always a polynomial, whence the first equivalence. As to the second equivalence, Theorem 2.6 clearly implies that $\phi *^{\prime} p=\phi * p$ iff $\sum_{\alpha \in 2 \pi Z^{s} \backslash 0} \phi *\left(e_{\alpha} p\right)=0$, yet this latter sum can vanish only if each of its summands vanishes, e.g., since the supports of the Fourier transforms of these summands are pairwise disjoint, and the sum converges in $\mathscr{S}^{\prime}$.

The following is a typical application of Corollary 2.8. Given a matrix $K_{s \times k}$, we employ the notation

$$
\nabla^{K}:=\prod_{\xi \in K} \nabla^{\xi}:=\prod_{\xi \in K}\left(1-E^{-\xi}\right)
$$

and record the following straightforward fact for use here and later.

Lemma 2.10. Let $\xi \in \mathbb{R}^{s}, \alpha \in \mathbb{C}^{s}$, and $q \in \Pi$. If $\nabla^{\xi}\left(e_{\alpha}\right)=0$ (equivalently, if $\left.e_{\alpha}(\xi)=1\right)$, then $\nabla^{\xi}\left(e_{\alpha} q\right)=e_{\alpha} \nabla^{\xi} q$. Otherwise, $\nabla^{\xi}$ is injective on $e_{\alpha} \Pi$. Consequently, for every translation invariant space $Q \subset \Pi, e_{\alpha} \nabla^{\xi} Q \subset \nabla^{\xi}\left(e_{\alpha} Q\right)$.

Corollary 2.11. Let $\phi$ be a compactly supported distribution. Then for any matrix $K_{s \times k}, \Pi\left(\nabla^{K} \phi\right) \subset \Pi(\phi)$.

Proof. Set $\psi:=\nabla^{K} \phi$. By [BR1], there exists a translation invariant $\mathcal{E} \subset \Pi$ such that $\psi *^{\prime} Q=\Pi(\psi)$. Since $\psi *^{\prime} Q \subset \Pi$, we may invoke Corollary 2.8 to obtain

$$
\phi *\left(\nabla^{K}\left(e_{\alpha} Q\right)\right)=\psi *\left(e_{\alpha} Q\right)=0, \quad \forall \alpha \in 2 \pi \mathbb{Z}^{s} \backslash 0 .
$$

But $Q$ is translation invariant, and therefore, by Lemma 2.10, $\phi *\left(e_{\alpha} \nabla^{K} Q\right) \subset$ $\phi *\left(\nabla^{K}\left(e_{\alpha} Q\right)\right)$. Thus,

$$
\phi *\left(e_{\alpha} \nabla^{K} Q\right)=0, \quad \forall \alpha \in 2 \pi \mathbb{Z}^{s} \backslash 0,
$$

which, together with Corollary 2.8 , implies that

$$
\phi *^{\prime} \nabla^{K} Q=\phi * \nabla^{K} Q=\left(\nabla^{K} \phi\right) * Q=\psi * Q=\psi *^{\prime} Q=\Pi(\psi) .
$$

Hence $\Pi(\psi) \subset \Pi(\phi)$ as claimed.

Remark 2.13. In case the matrix $K$ is integral, the preceding corollary becomes trivial, since then $S\left(\nabla^{K} \phi\right)=S(\phi)$, hence also $\Pi\left(\nabla^{K} \phi\right)=\Pi(\phi)$. However, the situation for a general $K$ is subtle, since the space $S\left(\nabla^{K} \phi\right)$ might be very different from $S(\phi)$. In particular, taking $\phi$ to be the characteristic function of the interval $[0,1)$, we check that $\Pi(\phi)=\Pi_{0}(\mathbb{R})$ while $\Pi\left(\left(1-E^{.5}\right) \phi\right)=\{0\}$, so that the inclusion in the corollary might be proper. Further, choosing $\phi$ to be the characteristic function of the interval $[0, .5)$, we get $\Pi(\phi)=\{0\}$, while $\Pi\left(\left(1+E^{-.5}\right) \phi\right)=\Pi_{0}(\mathbb{R})$, showing thereby that the corollary above does not extend to arbitrary difference operators (Lemma 2.10 does not carry over). 
Finally, note that no regularity assumption on $\phi$ has been made here, namely, the possibility $\hat{\phi}(0)=0$ has not been excluded.

\section{BOX SPLINES}

We say that $\phi$ provides a partition of unity if $1 \in S(\phi)$, i.e., if $\Pi(\phi)$ contains $\Pi_{0}$. Since $\Pi_{0} \subset \Pi(\phi) \Longleftrightarrow 1 \in \Pi(\phi)$, it follows from Proposition 2.2 and (2.4) that whenever $\hat{\phi}(0) \neq 0, \phi$ provides a partition of unity if and only if

$$
\phi *^{\prime} 1=\text { const. }
$$

Suppose now that $\Xi$ is an $s \times n$ matrix, and define

$$
\mathbb{K}_{U}(\Xi):=\left\{Y \subset \Xi: M_{\Xi \backslash Y} \text { does not provide a partition of unity }\right\} \text {. }
$$

We shall see later that the minimal elements of the set $\mathbb{K}_{U}(\Xi)$ can be determined directly from $\Xi$ without any recourse to box splines.

Theorem 3.2. For a matrix $\Xi_{s \times n}$,

$$
\Pi\left(M_{\Xi}\right)=\bigcap_{Y \in \mathbb{K}_{U}(\Xi)} \operatorname{ker} p_{Y}(D):=D_{U}(\Xi)
$$

We note that the theorem is trivial if $M_{\Xi}$ does not provide a partition of unity (since then $\Pi\left(M_{\Xi}\right)=\{0\}=D_{U}(\Xi)$ ). We may therefore assume that $\Pi_{0} \subset \Pi\left(M_{\Xi}\right)$; in particular, $\Xi$ is of rank $s$. Also, if $\Xi$ is an integral matrix and $V \subset \Xi$, then $M_{V}$ provides a partition of unity if and only if rank $V=s$ $[\mathrm{BH}]$. Thus Theorem 3.2 extends the result quoted in (1.10).

Roughly speaking, there are two different approaches toward the proof of the integral case of Theorem 3.2. One method [DM] is based on a clever calculation of the derivatives of the Fourier transform $\widehat{M}_{\Xi}$ on the lattice $2 \pi \mathbb{Z}^{s}$ (cf. [DM, Lemma 3.1, Theorem 3.1, and Proposition 3.2]). The other method [BH, BAR] is based on the identity [BH]

$$
p_{K}(D) M_{\Xi}=\nabla^{K} M_{\Xi \backslash K},
$$

which is valid for every $K \subset \Xi$. Both approaches extend to the nonintegral case. Here we exploit the latter method as a demonstration of the utility of Theorem 2.6 and Corollary 2.11. In the proof, we make use of the following simple lemmata.

Lemma 3.5. For every $K \in \mathbb{K}_{U}(\Xi), \Pi\left(M_{\Xi \backslash K}\right)=\{0\}$.

Proof. This follows directly from the definition of $\mathbb{K}_{U}(\Xi)$, the fact that $\Pi(\phi)$ is always translation invariant $[\mathrm{B}]$, and the fact that every nontrivial translation invariant polynomial space contains $\Pi_{0}$.

Lemma 3.6. Let $\xi \in \Xi$ and $\alpha \in \mathbb{C}^{s}$. Then

$$
M_{\xi} * e_{\alpha}=0 \Leftrightarrow\left(\nabla^{\xi} e_{\alpha}=0 \text { and } p_{\xi}(D) e_{\alpha} \neq 0\right) \text {. }
$$

Proof. The proof follows from the facts that

$$
\begin{gathered}
\nabla^{\xi} e_{\alpha}=0 \Leftrightarrow e_{\alpha}(-\xi)=1, \\
p_{\xi}(D) e_{\alpha} \neq 0 \Leftrightarrow p_{\xi}(\alpha) \neq 0, \\
M_{\xi} * e_{\alpha}=0 \Leftrightarrow \widehat{M}_{\xi}(\alpha)=\frac{e_{\alpha}(-\xi)-1}{-i p_{\xi}(\alpha)}=0,
\end{gathered}
$$


and the fact that the univariate entire function $e^{z}-1$ has only simple zeros.

Proof of Theorem 3.2. We first prove that

$$
\Pi\left(M_{\Xi}\right) \subset D_{U}(\Xi) \text {. }
$$

Since $\widehat{M}_{\Xi}(0)=1$, it suffices to show, in view of Proposition 2.2, that $M_{\Xi} *^{\prime} \Pi\left(M_{\Xi}\right) \subset D_{U}(\Xi)$. Let $p \in \Pi\left(M_{\Xi}\right)$ and $K \in \mathbb{K}_{U}(\Xi)$. By Proposition 2.2, $M_{\Xi} *^{\prime} p \in \Pi$ and, by (3.4),

$$
\Pi \ni p_{K}(D)\left(M_{\Xi} *^{\prime} p\right)=\left(\nabla^{K} M_{\Xi \backslash K}\right) *^{\prime} p \in S\left(\nabla^{K} M_{\Xi \backslash K}\right) \text {. }
$$

Hence, by Corollary 2.11 and Lemma 3.5,

$$
p_{K}(D)\left(M_{\Xi} *^{\prime} p\right) \in \Pi\left(M_{\Xi \backslash K}\right)=\{0\} .
$$

Thus, $M_{\Xi} *^{\prime} p \in \bigcap_{K \in \mathbb{K}_{U}(\Xi)} \operatorname{ker} p_{K}(D)=D_{U}(\Xi)$, as desired.

Next we prove that

$$
\Pi\left(M_{\Xi}\right) \supset D_{U}(\Xi) \text {. }
$$

Let $q \in D_{U}(\Xi)$. In view of Proposition 2.2 and Corollary 2.8 , it suffices to show that $M_{\Xi} *\left(e_{\alpha} q\right)=0$ for all $\alpha \in 2 \pi \mathbb{Z}^{s} \backslash 0$. To that end, fix $\alpha \in 2 \pi \mathbb{Z}^{s} \backslash 0$ and set

$$
K:=\left\{\xi \in \Xi: M_{\xi} * e_{\alpha}=0\right\}=\left\{\xi \in \Xi: \widehat{M}_{\xi}(\alpha)=0\right\} .
$$

We shall show that $M_{K} *\left(e_{\alpha} q\right)=0$, which would imply that $M_{\Xi} *\left(e_{\alpha} q\right)=0$ since $M_{\Xi}=M_{\Xi \backslash K} * M_{K}$. We first observe that (3.4) (with $\Xi$ there replaced by $K$ ) implies that for any $f \in \mathscr{D}^{\prime}\left(\mathbb{R}^{S}\right)$,

$$
\nabla^{K} f=M_{K} * p_{K}(D) f .
$$

Next we observe that $\widehat{M}_{\xi}(\alpha) \neq 0$ for $\xi \in \Xi \backslash K$, so $M_{\Xi \backslash K} * e_{\alpha}=e_{\alpha} \widehat{M}_{\Xi \backslash K}(\alpha) \neq 0$. By Corollary 2.8, $M_{\Xi \backslash K} *^{\prime} 1 \notin \Pi$, i.e., $K \in \mathbb{K}_{U}(\Xi)$. Consequently, $p_{K}(D) q=$ 0 , whence by (3.9),

$$
\nabla^{K} q=M_{K} * p_{K}(D) q=0 .
$$

As $M_{\xi} * e_{\alpha}=0$ for every $\xi \in K$, Lemma 3.6 allows us to deduce that $\nabla^{\xi} e_{\alpha}=0$. Hence, by Lemma 2.10 and (3.10), $\nabla^{K}\left(e_{\alpha} q\right)=e_{\alpha} \nabla^{K} q=0$. Appealing to (3.9) once again, we obtain

$$
p_{K}(D)\left(M_{K} *\left(e_{\alpha} q\right)\right)=\nabla^{K}\left(e_{\alpha} q\right)=0 .
$$

Finally, for every $\xi \in K, p_{\xi}(D) e_{\alpha} \neq 0$ by Lemma 2.10. This means that each $p_{\xi}(D), \xi \in K$, is $1-1$ on $e_{\alpha} \Pi$, hence so is $p_{K}(D)$. Thus, since $M_{K} *\left(e_{\alpha} q\right) \in$ $e_{\alpha} \Pi,(3.11)$ can hold only if $M_{K} *\left(e_{\alpha} q\right)=0$. This finishes the proof.

Theorem 3.2 reduces the computation of the approximation order of $S\left(M_{\Xi}\right)$ to the identification of those subsets $Y \subset \Xi$ for which $1 \in \Pi\left(M_{Y}\right)$. For an integral $\Xi$, we have already mentioned a simple criterion for this to hold, viz., $1 \in \Pi\left(M_{Y}\right)$ if and only if $Y$ is of rank $s$. However, for a general $\Xi$ the situation appears to be much more involved. We know of such a condition only when $s=1(1 \in \Pi(M \Xi)$ if and only if one of the entries of $\Xi$ is integral). The following example, whose computational details are omitted, indicates some 
of the difficulties that occur even in the bivariate setting. In what follows, we define

$$
\mathbb{K}_{U}^{\prime}(\Xi):=\left\{Y \in \mathbb{K}_{U}(\Xi): Y \backslash \xi \notin \mathbb{K}_{U}(\Xi) \text { for any } \xi \in Y\right\}
$$

and note that

$$
D_{U}(\Xi)=\bigcap_{Y \in \mathbb{K}_{U}^{\prime}(\Xi)} \operatorname{ker} p_{Y}(D) .
$$

Example 3.12. Let $s=2, n=8$, and

$$
\Xi=\left(\begin{array}{cccccccc}
\frac{1}{2} & 0 & \frac{1}{2} & 1 & \frac{1}{2} & \frac{1}{2} & \frac{1}{2} & 1 \\
0 & \frac{1}{2} & \frac{1}{2} & \frac{1}{2} & 1 & 1 & -\frac{1}{2} & 0
\end{array}\right) .
$$

Using the fact that for any $\xi \in \Xi$ and $\alpha \in \mathbb{Z}^{s}, \widehat{M}_{\xi}(2 \pi \alpha)=0$ if and only if $\xi \cdot \alpha \in \mathbb{Z} \backslash 0$, it can be verified that for $Y \subset \Xi, M_{Y}$ provides a partition of unity if and only if $Y$ contains one of the following matrices

$$
\begin{gathered}
Y_{1}:=\left(\begin{array}{cccccc}
\frac{1}{2} & 0 & \frac{1}{2} & 1 & \frac{1}{2} & \frac{1}{2} \\
0 & \frac{1}{2} & \frac{1}{2} & \frac{1}{2} & 1 & -\frac{1}{2}
\end{array}\right), \\
Y_{2}:=\left(\begin{array}{cc}
\frac{1}{2} & 1 \\
1 & 0
\end{array}\right) .
\end{gathered}
$$

It follows that the (six) elements of $\mathbb{K}_{U}^{\prime}(\Xi)$ are all $2 \times 2$, so Theorem 3.2 guarantees that $\Pi\left(M_{\Xi}\right)=\Pi_{1}$. The matrix $\Xi$ is irredundant in the sense that the removal of any column from $\Xi$ leads to a box spline $M$ with $\Pi(M) \neq \Pi_{1}$, yet one can remove five (!) columns from $\Xi$ to get

$$
\Xi^{\prime}:=\left(\begin{array}{ccc}
\frac{1}{2} & \frac{1}{2} & 1 \\
1 & 1 & 0
\end{array}\right)
$$

whose corresponding $\Pi\left(M_{\Xi^{\prime}}\right)$ is of dimension $2=\operatorname{dim} \Pi\left(M_{\Xi}\right)-1$. This is in stark contrast with the integral case, where the removal of any direction from $\Xi$ results in a corresponding polynomial space that is (strictly) smaller.

However, the approximation order of $S(M \Xi)$, for a general $\Xi$, can be computed as follows. First, for every $\alpha \in \mathbb{C}^{s}$, let $K_{\alpha}(\Xi):=\{\xi \in \Xi: \xi \cdot \alpha \in \mathbb{Z} \backslash 0\}$. Then we have

Theorem 3.13. Let $M \Xi$ be a box spline, and let $\left\{K_{\alpha}(\Xi): \alpha \in \mathbb{C}^{s}\right\}$ be as above. Then the approximation order from the space $S\left(M_{\Xi}\right)$ is the number

$$
\min \left\{\# K_{\alpha}(\Xi): \alpha \in \mathbb{Z}^{s} \backslash 0\right\} .
$$

Proof. From (1.2) it follows that

$$
\widehat{M}_{\xi}(2 \pi \alpha)=0 \Leftrightarrow \xi \in K_{\alpha}(\Xi),
$$

hence also

$$
M_{\xi} * e_{2 \pi \alpha}=0 \Leftrightarrow \xi \in K_{\alpha}(\Xi) .
$$

Therefore, for every $\alpha, M_{\Xi \backslash K_{\alpha}(\Xi)} * e_{2 \pi \alpha} \neq 0$, which implies, by Corollary 2.8, that $K_{\alpha}(\Xi) \in \mathbb{K}_{U}(\Xi)$ for every $\alpha \in \mathbb{Z}^{s} \backslash 0$. Furthermore, if $K \in \mathbb{K}_{U}^{\prime}(\Xi)$ then $1 \notin \Pi\left(M_{\Xi \backslash K}\right)$, hence, by Corollary 2.8, there exists an $\alpha \in \mathbb{Z}^{s} \backslash 0$ such that $M_{\Xi \backslash K} * e_{2 \pi \alpha} \neq 0$, which implies that $K_{\alpha}(\Xi) \subset K$; since both $K_{\alpha}(\Xi)$ and $K$ 
are elements of $\mathbb{K}_{U}(\Xi)$ and $K$ is minimal in $\mathbb{K}_{U}(\Xi)$, we get $K=K_{\alpha}(\Xi)$. We conclude that

$$
\mathbb{K}_{U}^{\prime}(\Xi) \subset\left\{K_{\alpha}(\Xi): \alpha \in \mathbb{Z}^{s} \backslash 0\right\} \subset \mathbb{K}_{U}(\Xi),
$$

hence

$$
D_{U}(\Xi)=\bigcap_{\alpha \in \mathbb{Z}^{s} \backslash 0} \operatorname{ker} p_{K_{\alpha}(\Xi)}(D)
$$

The required result now follows from Theorem 3.2 and the Strang-Fix Conditions.

\section{APPROXIMATION ORDER FROM SUBMODULE-TRANSLATES}

The determination of the approximation order of the box spline space $S\left(M_{\Xi}\right)$ admits an equivalent formulation, which, as a matter of fact, initiated our study here. This brief section is devoted to its discussion.

Let $\Xi$ be an $s \times n$ integral matrix and $\mathscr{A}$ a submodule of the $\mathbb{Z}$-module $\mathbb{Z}^{s}$ of length $s$, namely, $\mathscr{A}=A \mathbb{Z}^{s}$, where $A$ is an $s \times s$ integral matrix of full rank. Define

$$
S_{\mathscr{A}}\left(M_{\Xi}\right):=\operatorname{span}\left\{E^{\beta} M_{\Xi}: \beta \in \mathscr{A}\right\} .
$$

Proceeding as we did in the introductory section, we may define the approximation order of the space $S_{\mathscr{A}}\left(M_{\Xi}\right)$ in an entirely analogous fashion. Owing to the relation $[\mathrm{BH}]$

$$
M_{\Xi}=|\operatorname{det} A| M_{A} \Xi \circ A,
$$

the approximation order of $S_{\mathscr{A}}\left(M_{\Xi}\right)$ is seen to be precisely that of the spline space $S\left(M_{A^{-1} \Xi}\right)$. Therefore, Theorem 3.13 readily yields

Theorem 4.1. Let $\Xi$ be a $s \times n$ integral matrix, $A$ a $s \times s$ integral matrix of rank $s$, and $\mathscr{A}:=A \mathbb{Z}^{s}$. Then the approximation order from $S_{\mathscr{A}}\left(M_{\Xi}\right)$ is the number

$$
\min \left\{\# K_{\alpha}\left(A^{-1} \Xi\right): \alpha \in \mathbb{Z}^{s} \backslash 0\right\}=\min \left\{\# K_{\alpha}(\Xi): \alpha \in\left(A^{-1}\right)^{\mathrm{T}} \mathbb{Z}^{s} \backslash 0\right\} .
$$

Example 4.2. Let

$$
\Xi:=\left(\begin{array}{cccccccccccc}
1 & \cdots & 1 & 0 & \cdots & 0 & 1 & \cdots & 1 & 1 & \cdots & 1 \\
0 & \cdots & 0 & 1 & \cdots & 1 & 1 & \cdots & 1 & -1 & \cdots & -1
\end{array}\right),
$$

where the vectors $\xi_{1}:=(1,0), \xi_{2}:=(0,1), \xi_{3}:=(1,1)$, and $\xi_{4}:=(1,-1)$ occur with multiplicities $n_{1}, n_{2}, n_{3}$, and $n_{4}$, respectively. Let

$$
A:=\left(\begin{array}{cc}
1 & 1 \\
1 & -1
\end{array}\right) \quad \text { and } \quad \mathscr{A}:=A \mathbb{Z}^{2}
$$

so that

$$
\left(A^{-1}\right)^{\mathrm{T}} \mathbb{Z}^{2}=\left\{\alpha=\left(\alpha_{1}, \alpha_{2}\right) \in \frac{1}{2} \mathbb{Z}^{2}:\left(\alpha_{1}+\alpha_{2}\right) \in \mathbb{Z}\right\} .
$$

Suppose that $\alpha \in\left(A^{-1}\right)^{\mathrm{T}} \mathbb{Z}^{2} \backslash 0$. Then $\alpha \cdot \xi_{j} \in \mathbb{Z}, j=3,4$, so $\# K_{\alpha}(\Xi) \geq n_{3}+n_{4}$ unless one of $\alpha \cdot \xi_{3}$ or $\alpha \cdot \xi_{4}$ is zero; in which case $\# K_{\alpha}(\Xi) \geq \min \left\{n_{3}, n_{4}\right\}$. In fact, choosing $\alpha=\left(\frac{1}{2}, \pm \frac{1}{2}\right)$, we see that $\min \left\{\# K_{\alpha}(\Xi): \alpha \in\left(A^{-1}\right)^{\mathrm{T}} \mathbb{Z}^{2} \backslash 0\right\}=$ $\min \left\{n_{3}, n_{4}\right\}$. As a result, Theorem 4.1 implies that the approximation order of $S_{\mathscr{A}}\left(M_{\Xi}\right)$ is $\min \left\{n_{3}, n_{4}\right\}$. We thus recover [JR, Theorem 3]. It is not without interest to note, that in view of (1.12), the approximation order of $S\left(M_{\Xi}\right)$ (as opposed to $\left.S_{\mathscr{A}}\left(M_{\Xi}\right)\right)$ is $\min \left\{-n_{j}+\sum_{k=1}^{4} n_{k}: j=1,2,3,4\right\}$. 


\section{REFERENCES}

[B] C. de Boor, The polynomials in the linear span of integer translates of a compactly supported function, Constr. Approx. 3 (1987), 199-208.

[BAR] A. Ben-Artzi and A. Ron, Translates of exponential box splines and their related spaces, Trans. Amer. Math. Soc. 309 (1988), 683-710.

[BH] C. de Boor and K. Höllig, B-splines from parallelepipeds, J. Analyse Math. 42 (1982/3), 99-115.

[BHR] C. de Boor, K. Höllig, and S. D. Riemenschneider, Box splines, Springer-Verlag, Berlin and New York, 1992.

[BR1] C. de Boor and A. Ron, Polynomial ideals and multivariate splines, Multivariate Approximation Theory V (W. Schempp and K. Zeller, eds.), Birkhäuser, Basel, 1990, pp. 31-40.

[BR2] _- The exponentials in the span of the integer translates of a compactly supported function: approximation order and quasi-interpolation, J. London Math. Soc. (2) (to appear).

[C] C. K. Chui, Multivariate splines, CBMS-NSF Regional Conf. Ser. Appl. Math., vol. 54, SIAM, Philadelphia, 1988.

[DM] W. Dahmen and C. A. Micchelli, Translates of multivariate splines, Linear Algebra Appl. 52/3 (1983), 217-234.

[F] H. G. Friedlander, Introduction to the theory of distributions, Cambridge Univ. Press, London and New York, 1982.

[JR] K. Jetter and S. D. Riemenschneider, Cardinal interpolation with box splines on submodules of $\mathbb{Z}^{d}$, Approximation Theory V (C. K. Chui, L. L. Schumaker, and J. D. Ward, eds.), Academic Press, New York, 1986, pp. 403-406.

[R] A. Ron, A characterization of the approximation order of multivariate spline spaces, Studia Math. 98 (1991), 73-90.

[SF] G. Strang and G. Fix, A Fourier analysis of the finite element variational method, C.I.M.E. II Cilo 1971, Constructive Aspects of Functional Analysis (G. Geymonet, ed.), 1973, pp. 793-840.

Center for the Mathematical Sciences, University of Wisconsin-Madison, Madison, WISCONSIN 53705

E-mail address: amos@cs.wisc.edu

Department of Mathematics, University of Alberta, Edmonton, Alberta, Canada T6G 2G1

Current address: Department of Mathematics, Center for Approximation Theory, Texas A\&M

University, College Station, Texas 77843-3368

E-mail address: nob9437@tamvenus.bitnet 